\title{
Meta Managed Objects
}

\author{
J. Seitz \\ Institute of Telematics \\ University of Karlsruhe \\ Germany \\ Phone +497216084020 \\ Fax +49721388097 \\ E-Mail seitz@telematik.informatik.uni-karlsruhe.de
}

\begin{abstract}
Network management has become a crucial affair in today's business. This task needs computer assistance due to the complexity and dimension of the networks. But this computer assistance has to cope with a problem, that has been running through the history of computer communication: due to the lack of one standard in network management heterogeneous management protocols and management models have emerged. In order to manage a large computer network several management standards have to be considered.

To avoid dealing with different management application that can only manage a certain part of the whole network a way of integrating the different management worlds has to be achieved. This paper explains a way to integrate different network models by introducing so called Meta Managed Objects. The described work is part of a project carried through at the University of Karlsruhe [Sei94a].
\end{abstract}

\section{Keywords}

Network management model, integration of management architectures

\section{MOTIVATION}

Today's business rely heavily on communication and cooperation services provided by computer networks. Failures in these networks can lead to fatal consequences. Thus, network planning is a crucial task and when running the network bottlenecks have to be identified and failures have to be located before they lead to unbearable problems. Of course, all these tasks of network planning and network management must be computer-aided, since the complexity and the dimensions of today's networks make the network operator reach his limits soon when trying to cope with the problems by himself.

In order to carefully plan and to comprehensively manage computer networks, certain standards have evolved dealing with the way to define information needed for these tasks and to have access to this information. Besides proprietary management solutions like IBM's NetView for their SNA networks, two different management worlds have emerged: on the one hand there is a management framework defined by the ISO for their Open Systems Interconnection (OSI), on the other hand the Internet 
Society has developed the Simple Network Management Protocol SNMP with its own management model. Although SNMP was thought as an transitional solution it has proved to be practical and has therefore been extended to overcome its weaknesses due to its simplicity.

Nevertheless, there is no hint on one and only future management standard. All the standards will coexist and there are different markets for them. There is no substitute for SNMP and its successors in the area of managing and planning Local Area Networks, while the ISO management framework is mainly applied by the carriers of public and wide areas networks, especially in Europe. But with the interworking between local and public networks there will always be the problem to deal with both standards at the same time. Let us have a look at the ATM management model as defined by the ATM Forum [AC95] (cf. Figure 1). There are five management interfaces: M1 and M2 define the interface between the customer's management system and the ATM end-station. M3 is the Customer Network Management Interface. With M4 the interface between private and public networking technologies is defined. Finally, M5 is the interface between different Carrier Network Management Systems.

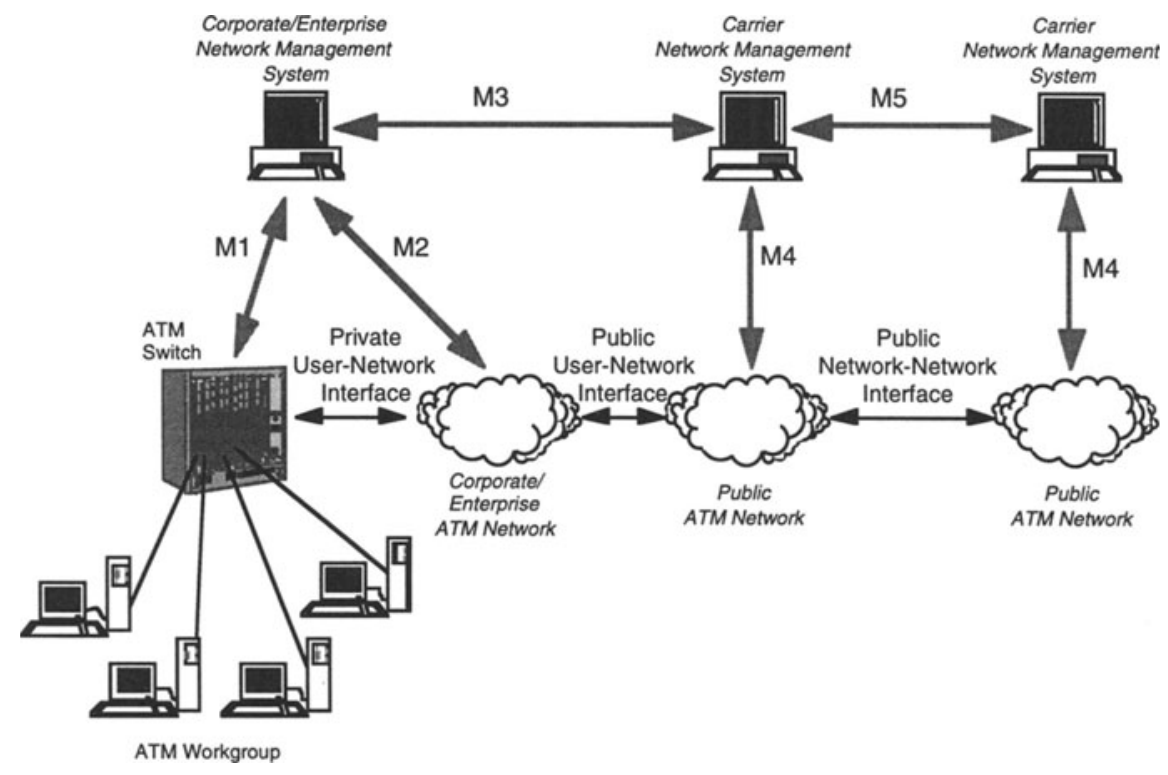

Figure 1 The ATM Management Model.

Obviously, at the public user-network interface the two management worlds meet. But that means, that the (usually SNMP-based) corporate/enterprise network management system tries to get information out of components based on the ISO management framework. For the carrier network management system the problem is vice versa. Thus, the management application has to make a difference between the ways to have access to the management information according to the supported management architecture. But this would lead to very complex and voluminous implementations. To overcome this situation different approaches have been worked out that will be introduced in section 2. Section 3 details the concepts of so called Meta Managed Objects, that integrate different management models. The practicability of this approach is described in section 4 by a simple but comprehensible example. Finally, section 5 concludes this paper with some aspects that are deeply associated with the 
concepts of Meta Managed Objects but could not be described in detail due to the spatial limitations of this paper.

\section{DIFFERENT APPROACHES TO INTEGRATED NETWORK MANAGEMENT}

Let us first consider the two management worlds. Although there is the opinion, that »any API that tries to be management protocol independent will capture the worst of both worlds « [Ros93], many approaches have been made in order to make these worlds meet. There are different ways to approach the goal of integrating the management worlds:

- First, management agents have been developed that are independent of the used management protocols, i.e., they support different management protocols [MBL92]. Thus, any management application may retrieve information from this agent independently of the used management protocol. However, these agents are very complex and afford plenty of processor and memory capacity. Thus, they are not practicable for network elements that are limited in memory and computing performance.

- Second, there is the solution of implementing a management gateway between the different worlds. These gateways are also called management proxies [Cha93], [Gre93] and work usually only one-way, i.e., they support management applications based on one management protocol and agents based on another. Besides, these proxies usually support a MIB especially designed for the management information found in the hidden management agents. Thus, they are not very flexible and the management application must differentiate, whether it addresses an agent based on the same management protocol or a heterogeneous agent via the proxy.

- Third, management platforms have evolved dealing with more than one management architecture. E.g., the Distributed Management Environment (DME) as defined by the Open Software Foundation (OSF) [OSF92] supplies the traditional management framework based on OSI and internet management and has added an object-oriented framework using the common object request broker architecture (CORBA). Nevertheless, this architecture has proven to be very difficult to implement [Mar94], that indeed no implementation is available covering all the architecture's aspects. Another example for an integrating management platform is Hewlett-Packard's OpenView AdminCenter [HP94] using SNMP, RPC and CORBA to have access to management information. There they use special model languages to integrate different management models based on SMALLTALK.

Although these solutions may all help to integrate the different management worlds, they still lack in some points. The next section will discuss their disadvantages and show another way of integrating different management protocols, namely the concept of Meta Managed Objects.

\section{THE CONCEPT OF META MANAGED OBJECTS}

The described approaches have different disadvantages:

- Whenever a new management architecture comes up, all the transforming entities, i.e., the management gateways or the protocol independent agents, have to be reimplemented. The same occurs whenever there are new managed objects to deal with. 
- On the other hand, there is new overhead produced: with the management gateways you have some machines doing the transformation of management protocols and information and with the protocol independent agents the agent's code is so voluminous that it might not work on network elements with little memory such as bridges or hubs.

So the best would be a flexible interpretable approach as it is realized by the concept of Meta Managed Objects. Therefore, a Meta Managed Object (cf. Code Segment 1) consists of

- the object's name in order to instantiate the object;

- the object's access rights;

- a list of the object's attributes to which we will come later on;

- a list of possible operations that can be performed on this object;

- a list of reports the object can send out whenever some critical or exceptional situation has occurred.

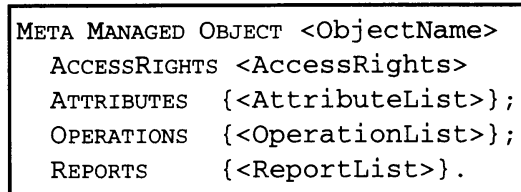

Code Segment 1 Definition of a Meta Managed Objects.

The integration of different management models within these Meta Managed Objects is achieved by correlating items of these models in the lists of attributes, operations or reports as mentioned above. Thus, let us have a closer look at the way an object's attribute is described (cf. Code Segment 2). This attribute is definitely identified by a name (after the key word ATTRIBUTE), its type is determined by the key word TYPE and its access rights are defined by the key word ACCESS. Then there is a correlation list containing the following information:

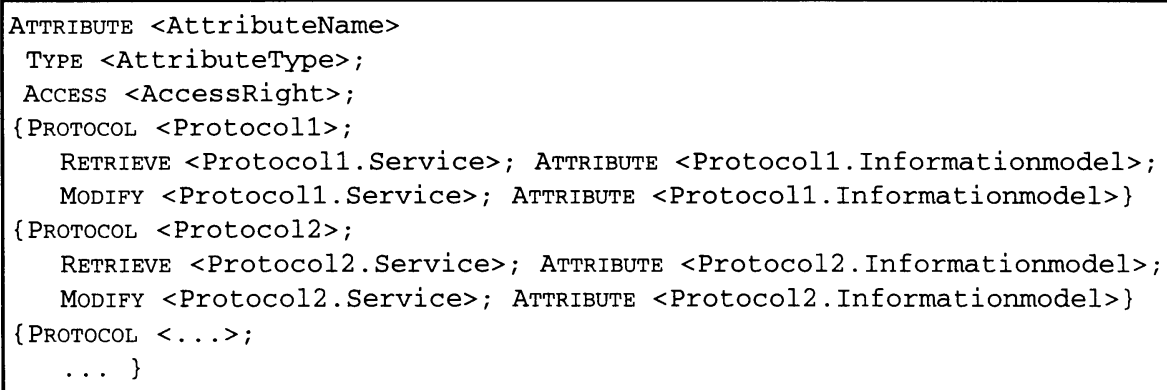

Code Segment 2 Definition of an attribute within a Meta Managed Object.

- With the key word PROTOCOL the management protocol to which the following information relate is defined.

- The statement with the key word RETRIEVE describes the service of the management protocol to use in order to have access to the management information 
corresponding to this attribute.

- With the key word ATTRIBUTE is defined where to find the corresponding information in the management model when using the determined management protocol. Since there might not be only a 1:1 relation between attributes it is possible to define a computation of the attribute's value by giving a numerical expression consisting of arithmetical operations and values defined in the management model. E.g., the attribute's value corresponds to the sum of $x$ and $y$ divided by 2 , whereby $\mathrm{x}$ and $\mathrm{y}$ are managed objects or attributes of managed objects in the management model of the management protocol defined with the key word PROTOCOL.

- In the next line the key word MODIFY leads to the management protocol service you have to use in order to modify the management information.

- Again the key word ATTRIBUTE gives the hint to the corresponding information in the management model on which the determined management protocol is based on. It is necessary to differentiate between reading and modifying the information, because if there is not only one management item concerned (cf. point 2) you must define how a modification reflects on the values of the different management items.

The operations of a Meta Managed Object can be found in a list of operations. Each operation is defined in terms of its name (unique within the Meta Managed Object) and the different ways to perform this operation according to the different management protocols and models. Therefore, for each management protocol the operation and the concerned attributes are listed (cf. Code Segment 3).

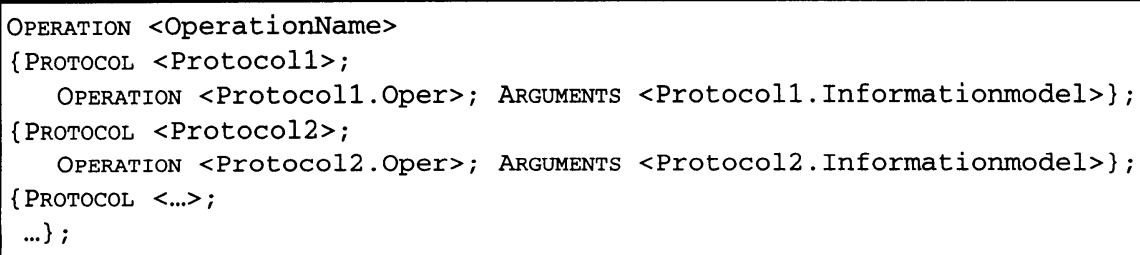

Code Segment 3 Definition of an operation within a Meta Managed Object.

Finally, the Meta Managed Object contains a list of reports, too, that might be received whenever an exceptional situation occurs. Since management agents based on different management protocols use different means to report such a situation (e.g. via traps in SNMP or via event reports in CMIS/P), the received report and the report that the user expects have to be mapped. This mapping is done in the report definition, where the report is identified by a name, where the report's output values are specified, and where the report is correlated to the possible reports in other management models (cf. Code Segment 4).

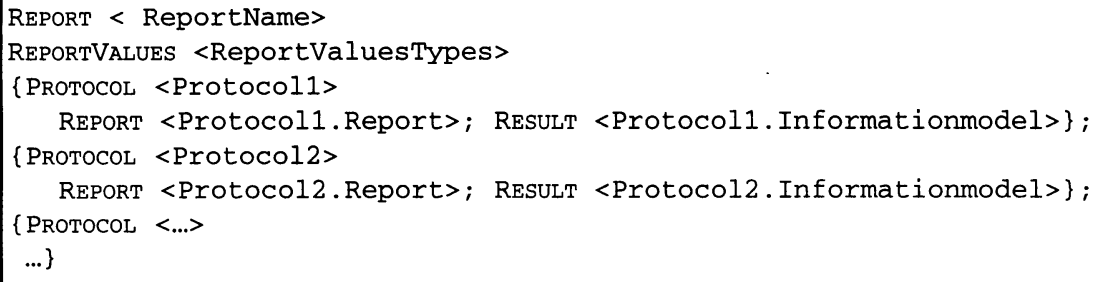

Code Segment 4 Definition of a report within a Meta Managed Object. 
Of course, you cannot presuppose that there is always a mapping between different management models. There will always be discrepancies between the models leading to some lines in the definition of a Meta Managed Object not to be completed. Therefore, the value Null can be specified to point out that this information cannot be found in the concerned management model. If an attribute of a Meta Managed Object might result from a sum of two attributes found in a specific management model, it is likely that a modification of the Meta Managed Object's attribute cannot be performed within the given management model.

Anyway, a correlation between different management models has been achieved. Nevertheless, this is only a model correlation, that must be instantiated according to the managed object classes being instantiated in managed objects. There are two cases to be detailed:

If there is only one instance per managed element, then the instantation is simple. In order to identify the instance the Meta Managed Object's name an the agent's address are concatenated. Then there is a list of pairs mapping the Meta Managed Object's attributes with attributes in the agent's MIB, the Meta Managed Object's operations with the operations supported by the agent and the Meta Managed Object's reports with the event reports or the traps the agent is able to send out.

The second case is somewhat more complex. The Meta Managed Object might be instantiated several times per managed element. Thus the identifiers for the instances must be extended by a number that has to be incremented whenever a new instance is created or has been encountered. Then again, the Meta Managed Object's attributes, operations, and reports have to be mapped within lists. Let us have a closer look on how the instances might be created: the application might ask for all the instances of a Meta Managed Object. Then, the addressed agent is accessed (by using the supported management protocol) and all the values of the objects or attributes, operations and reports in the agent's MIB that appear in the Meta Managed Object's description are requested. The results are then ordered (e.g. combining the attributes of an ISO/OSI managed object or gathering the attributes of a row in an SNMP-table) to form the instances of the Meta Managed Object giving them unique identifiers as described above.

Thus, there is not only a mapping of the different management models, but also a correlation of the managed objects to be supported by an agent. The following section will detail this procedure by giving an example. This might appear rather simple but in order to concentrate on the core of the described approach it can explain its functioning.

\section{EXAMPLE OF A META MANAGED OBJECT}

In order to illustrate the concept of Meta Managed Objects let us have a look at an example concerning systems management. In a local area network the system administrator wants to generate a login procedure that chooses the machine to log on depending on the momentary number of users on that machine. Thus, he tries to equally load the machines he is responsible for. To make the problem more complex, the network consists of heterogeneous machines that support different management models. To retrieve the desired information, i.e., the number of users logged on, the login procedure has to take care of the supported management models what makes the implementation very complex and inflexible.

The solution would be the introduction of Meta Managed Objects. Therefore, let use assume the following three management models supplying the information about the momentary number of users:

1. In the „Host Resources MIB“ [GW93] there is the Managed Object hrSystemNumUsers, that supplies the desired information: 


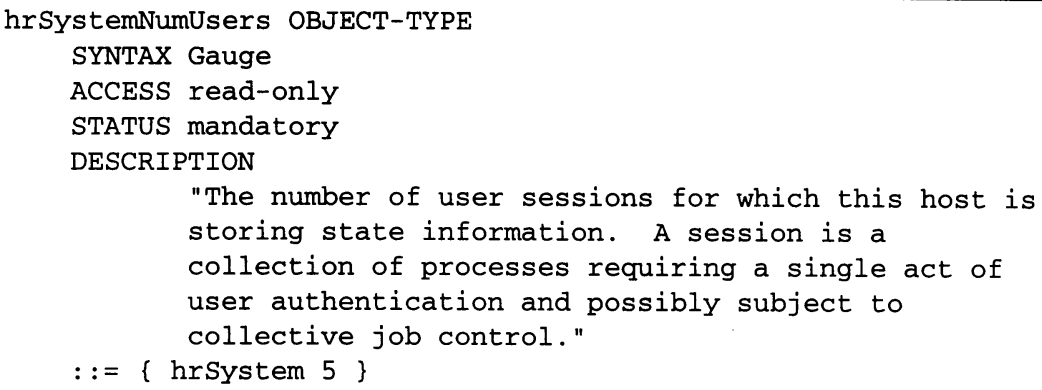

Code Segment 5 SNMP managed object hrSystemNumUsers.

2. If the information is supplied by an ISO/OSI management agent, it might implement the Management Information Base according to the OSIMIS (OSI Management Information Service) [UCL92] based on the ISO-Development Environment ISODE (to simplify the definition of this object we have concentrated on the GDMO-definition of a package for this object, that is a collection of attributes and notifications contained in the object) :

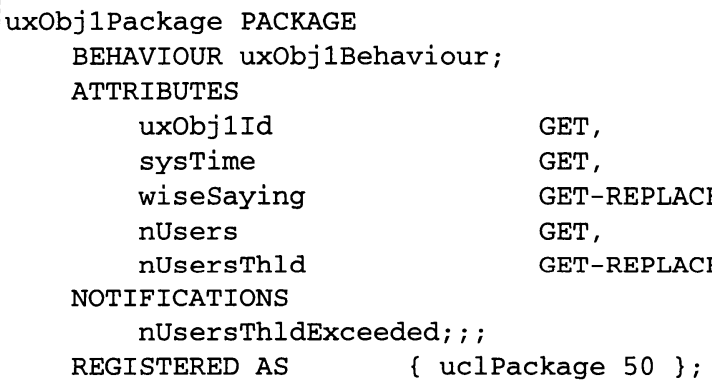

Code Segment 6 ISO/OSI managed object package uxObj1Package.

3. Finally, if the machine does not supply any management agent at all, there is still the possibility to get the desired information via a UNIX system call (who) and counting the number of entries:

rsh <node> 'who | wc -1'

Code Segment 7 UNIX system call.

These three possibilities to access and to model the information might be integrated into one Meta Managed Object: 


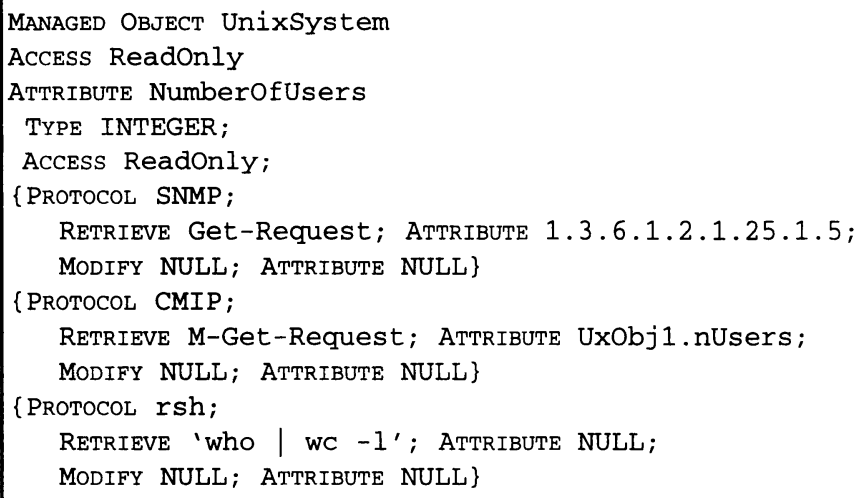

Code Segment 8 Meta Managed Object UnixSystem.

Of course, one can add the information needed to report the machine being overloaded by users. In Code Segment 6 there is the notification nUsersThldExceeded defined, that is sent out, whenever the number of users exceeds a given threshold. The same effect could have an SNMP-trap, if it is defined in the SNMP-MIB. Thus, adding a report definition to the Meta Managed Object would extend its functionality.

Obviously, this approach is very flexible. Whenever a new management protocol or even a new way of accessing the desired information comes up, the Meta Managed Object has to be extended by only the statements of the new protocol and the relevant services and attributes to retrieve and to modify the information.

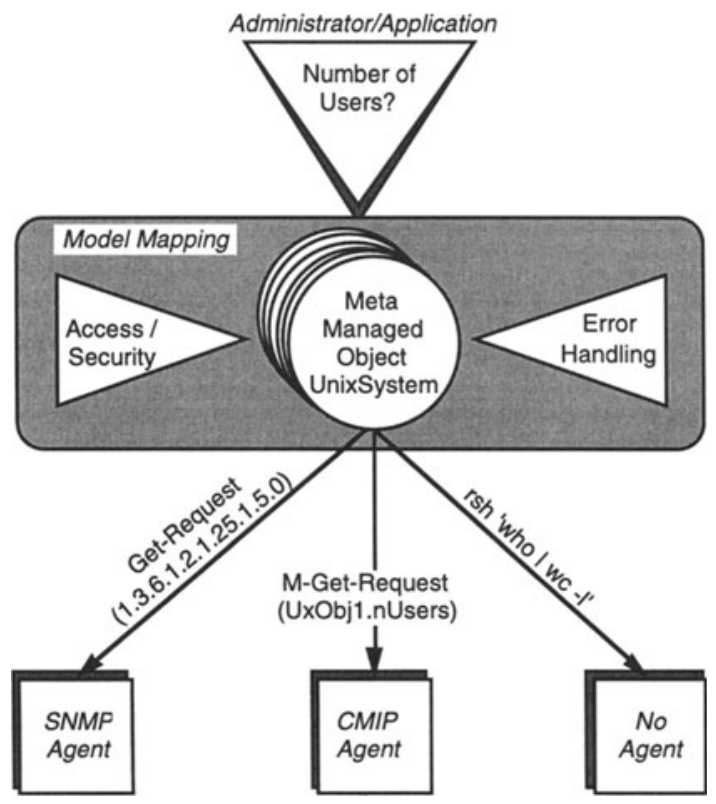

Figure 2 Illustration of the exemplary Meta Managed Object. 
Figure 2 describes the example. The different ways to get to the information are transparently hidden by the concept of Meta Managed Objects. The user respectively the application is simply asking for the number of users from a specified node and gets an INTEGER as the answer.

\section{CONCLUSION}

The described approach of Meta Managed Objects is a way of integrating different management models. It is very flexible since it is interpreted and dynamically adopted to the instances found in the addressed management agents. Nevertheless, the Meta Managed Objects are only one aspect of an integrating management architecture. Further items to bear in mind are:

1. the integration of management protocols:

Since not only the management models but also the management protocols differ, a way to integrate these protocols has to be worked out. Together with the Meta Managed Objects a Generic Management Protocol GMP has been defined [Sei94b], that maps the services of any management protocol onto the GMPservices and vice versa. Thus, e.g., one can request an SNMP-service, that is then transformed into a CMIP-request by using GMP as an intermediate protocol. The answer is then re-transformed into an SNMP-response.

2. the interpretation of error messages:

Just like management models and management protocols differ, they also send out different error massages. Thus, these error messages must be mapped as well in order to avoid misunderstandings. For example, if a CMIP-M-Get-Request has resulted in an error message ,noSuchAttribute“, a SNMP-based application would expect to receive an error message „,noSuchName“.

3. the integration of security mechanisms:

Network management is a very critical tasks. With wrong operations you will not only not remove the reported network problem but cause a total net breakdown. Thus, only authorized users and/or applications may change management information. To make sure that authentication and authorization work, security mechanisms have to be provided. Nevertheless, there are different security levels in different management architectures. An integration of these security mechanisms provided by different management standards has to be achieved so that a management application can choose the level of security it needs to carry through its management task.

The co-working of the described mapping mechanisms is presented in [Sei94a]. The architecture has been implemented as a prototype and has proven its practicability in an AI-based network management tool, that has been developed since 1991 [Sei91], [Röt92], [SSS96]. Anyway, the architecture has not been tuned in items of speed or throughput, but it worked fine with a non-time-crucial expert system.

The future work is focused on the management of agent-based distributed systems using the described architecture [SS95]. Furtheron, the architecture is adopted to the task of tariffing broadband services. As already mentioned in section 1, especially with ATM the different management worlds will meet needing integrated management solutions. 


\section{REFERENCES}

[AC95] P. Alexander and K. Carpenter. ATM Net Management: A Status Report. Data Communications International, Vol. 24, No. 12, September 1995, pp. $110-116$.

[Cha93] A. Chang: ISO/CCITT to Internet Management Proxy. Internet Draft, March 1993.

[Gre93] U. Gremmelmaier: The Internet Proxy Agent - A Link between OSI and TCP/IP Management. In Conference Proceedings of the Third Joint Workshop on High Speed Networks, Paris, France, March 1993.

[GW93] P. Grillo and S. Waldbusser: Host Resource MIB. Request for Comments 1514. Network Working Group, September 1993.

[HP94] Hewlett-Packard GmbH, Network \& System Management Division: HP OpenView Admin Center. External Specification, Böblingen, 1994.

[Mar94] J.S. Marcus: Icaros, Alice and the OSF DME. In Proceedings of the Fifth IFIP/IEEE International Workshop on Distributed Systems: Operations \& Management (DSOM'94), October 10-12, 1994, Toulouse, France, pp. 4.1-4.21.

[MBL92] S. Mazumdar, S. Brady, and D.W. Levine: Design of Protocol Independent Management Agent to Support SNMP and CMIP Queries. Research Report RC 18246 (80062), IBM Research Division, T.J. Watson Research Center, Yorktown Heights, NY, USA, August 13, 1992.

[OSF92] The Open Software Foundation: OSF Distributed Management Environment (DME) Architecture. May 1992.

[Röt92] J. Röthig: Considerations on the Choice of Bandwidth Balancing Moduli in DQDB Metropolitan Area Networks. In Proceedings of European Optical Communications and Networks - Tenth Annual EFOC/LAN ' 92 Conference, Papers on Networks, Paris, France, June 24-26, 1992, pp. 235-240.

[Ros93] M.T. Rose: Network Management: Status and Challenges. Connexions The Interoperability Report, Vol. 7, No. 6, June 1993, pp. 11-17.

[Sei91] J. Seitz: An Architecture for an Expert System Aiding in Network Planning and Network Management. In K. Majithia (ed.): Silicon Valley Networking Conference SVNC, Proceedings, Santa Clara, CA, USA, April 1991, Maple Press pp. 197-202.

[Sei94a] J. Seitz: Integration heterogener Netzwerkmanagementarchitekturen. No. 289, Series 10: Computer Science/Communications Technology, VDIVerlag, Düsseldorf, 1994 (German).

[Sei94b] J. Seitz: Towards Integrated Network Management: A Generic Management Protocol. In Proceedings of the Fifth IFIP/IEEE International Workshop on Distributed Systems: Operations \& Management (DSOM'94), October 10-12, 1994, Toulouse, France, pp. 6.1-6.18.

[SS95] G. Schäfer and J. Seitz: Managing Agent-Systems: A Key Issue for Dealing with Complexity. In Proceedings of the Intelligent Agents Workshop, Oxford Brookes University, Oxford, England, November 23, 1995, pp. 85-86. 
[SSS96] G. Schäfer, J. Schiller, and J. Seitz: A Toolkit for Rapid Prototyping of Expert Systems for Integrated Network Management. In M.H. Hamza (ed.): Proceedings of the Fourteenth IASTED International Conference „Applied Informatics“, Innsbruck, Austria, February 20-22, 1996, pp. 6567.

[UCL92] A Guide to Implementing Managed Objects Using the GMS. Universal College London. Version 2.99.1/Draft 1, October 1992.

\section{BIOGRAPHY}

Jochen Seitz has studied Computer Science at the University of Karlsruhe in Germany. Then, he joined the University's Institute of Telematics in 1989, where he finished his $\mathrm{Ph} . \mathrm{D}$. thesis in 1993. He is still with the institute, preparing courses on telematics and working in the area of application, network and systems management. He is mainly interested in the areas of security and accounting management. 